\title{
A generalization of the Shapley-Ichiishi result
}

\author{
Jeroen Kuipers • Dries Vermeulen • \\ Mark Voorneveld
}

\begin{abstract}
The Shapley-Ichiishi result states that a game is convex if and only if the convex hull of marginal vectors equals the core. In this paper, we generalize this result by distinguishing equivalence classes of balanced games that share the same core structure. We then associate a system of linear inequalities with each equivalence class, and we show that the system defines the class. Application of this general theorem to the class of convex games yields an alternative proof of the Shapley-Ichiishi result. Other applications range from computation of stable sets in non-cooperative game theory to determination of classes of TU games on which the core correspondence is additive (even linear). For the case of convex games we prove that the theorem provides the minimal defining system of linear inequalities. An example shows that this is not necessarily true for other equivalence classes of balanced games.
\end{abstract}

Keywords TU games · Core $\cdot$ Linearity regions $\cdot$ Computation of Q-sets

\footnotetext{
J. Kuipers $(\varangle)$

Department of Knowledge Engineering, Maastricht University, P.O.Box 616, 6200 MD Maastricht, The Netherlands

e-mail: kuipers@maastrichtuniversity.nl

D. Vermeulen

Department of Quantitative Economics, Maastricht University, P.O.Box 616, 6200 MD Maastricht, The Netherlands

e-mail: d.vermeulen@maastrichtuniversity.nl

M. Voorneveld

Department of Economics, Stockholm School of Economics, P.O.Box 6501, 11383 Stockholm, Sweden e-mail: Mark.Voorneveld@hhs.se
} 


\section{Introduction}

In his study of convex games, Shapley (1971) proved that the core of a convex game is the convex hull of the marginal vectors of that game. A decade later, Ichiishi (1981) showed that the converse of this statement is also true: if the core of a game is equal to the convex hull of marginal vectors, then the game is convex. The combined ShapleyIchiishi result thus relates the defining system of linear inequalities of convex games to the combinatorial structure of the core common to all convex games. The interesting combinatorial properties of convex games has led to a vast literature in which convex games play a role. Examples are unanimity games, bankruptcy games (O’Neill 1982; Aumann and Maschler 1985), sequencing games (Curiel et al. 1989), standard tree games (Megiddo 1978, Granot et al. 1996), and extended tree games (Granot et al. 2002). The Shapley-Ichiishi result is used freely in the analysis of such games.

In this paper, we generalize the Shapley-Ichiishi result to other classes of balanced games. Specifically, we distinguish equivalence classes of balanced games based on the notion of core structure. The core structure of a given balanced game is a collection of subsets of coalitions of the game such that each member of this collection corresponds exactly to the set of binding constraints at some extreme point of the core of the balanced game under consideration. Two balanced games are considered to be equivalent when they have the same core structure. We provide a procedure which, given an equivalence class with respect to this equivalence relation, generates a defining system of linear inequalities that describes the closure of the class. One of the equivalence classes is the class of strictly convex games, and its closure is the class of convex games.

Our result has several applications. A first application is analysis of the core correspondence. As is well known, the core correspondence is superadditive. The equivalence classes defined in this paper are exactly those sets of balanced games on which the core is precisely additive. These sets are determined by the linear inequalities presented in our main theorem. ${ }^{1}$

A second application is in the context of stability of Nash equilibrium for bimatrix games. The main theorem of our paper can be used to construct a finite algorithm to determine whether a set of strategy pairs in a given bimatrix game contains a Q-set as defined in Vermeulen et al. (1996). Details of this construction are discussed in the conclusion of this paper.

A third application of the generalized Shapley-Ichiishi result is the analysis of certain classes of games defined by linear inequalities, for example the class of $k$-convex games. It is known that these games are balanced and that a full characterization of the extreme core elements can be given (Driessen 1986). Indeed, this characterization implies that all $k$-convex games share the same core structure. Therefore, the main theorem of our paper also applies to the class of $k$-convex games.

The setup of this paper is as follows. In Sect. 2, we define the core structure of a game, which gives rise to equivalence classes of games with the same core structure, and we describe the core structure of convex games. In Sect. 3, we give a short review

\footnotetext{
1 Our result extends to analysis of the correspondence $b \mapsto \varphi(b)$, where $\varphi(b)$ is the solution set of a system $A x \geq b$ of linear inequalities.
} 
on polyhedra and their facial structure. In Sect. 4, we provide a constructive method for generating a system of linear inequalities associated with a given core structure, and we show that the system defines the closure of the equivalence class. An example shows that the system may contain redundant inequalities. From the description with inequalities, it follows that the closure is a polyhedral cone. These cones are of interest from a computational perspective, as it can be shown that the core is an additive correspondence on each cone. In Sect. 5, we apply our main theorem to the class of convex games, and this way, we derive an alternative proof of the Shapley-Ichiishi result. In fact, we obtain a somewhat sharper result, since the set of defining inequalities that we obtain turns out to be a strict subset of the set of inequalities that is normally used to define convexity. We then prove that each inequality in our system describes a facet of the cone of convex games. Hence, in the case of convex games, we obtain the minimal system. In the conclusion we discuss our main result and its consequences.

\section{Games and their core structure}

A transferable utility game, or game, is a pair $(N, v)$ where $N=\{1, \ldots, n\}$ is the set of players and $v$ is a function that assigns to each coalition $S \subseteq N$ its worth $v(S) \in \mathbb{R}$. The worth $v(\phi)$ of the empty set is zero. Throughout this paper we keep the player set $N$ fixed. So we simplify notation and write $v$ instead of $(N, v)$ to denote a game.

A vector $x \in \mathbb{R}^{N}$ is an allocation. The $i$ th coordinate $x_{i}$ of the allocation $x$ represents the payoff to player $i \in N$. For coalition $S \subseteq N$, the aggregate payoff $\sum_{i \in S} x_{i}$ is denoted by $x(S)$. An allocation $x$ is efficient for $v$ if it distributes the worth of the grand coalition among the players of the game $v$, i.e., if $x(N)=v(N)$. An efficient allocation $x$ is a core allocation for $v$ if

$$
x(S) \geq v(S) \text { for all } S \subset N .
$$

The set of core allocations, denoted by $C(v)$, is called the core of $v$. A game whose core is not empty is balanced.

A nonempty coalition $S$ with $x(S)=v(S)$ is called tight at $x$ in $v$. The collection of coalitions that are tight at $x$ in $v$ is denoted by $T(v, x)$. Define the core structure of $v$ by

$$
\mathcal{T}(v):=\{T(v, x) \mid x \text { is an extreme point of } C(v)\} .
$$

We say that $w$ is a limit game for $v$ if for every extreme point $x \in C(v)$ there exists an extreme point $y \in C(w)$ such that $T(v, x) \subseteq T(w, y)$. The collection of limit games for $v$ is denoted by $L(v)$.

In the remaining part of this section, we focus on convex games and their core structure. A game $v$ is convex if it satisfies the inequalities

$$
v(S)+v(T) \leq v(S \cap T)+v(S \cup T)
$$

for all coalitions $S$ and $T$. If the inequality in (*) is strict whenever $S \nsubseteq T$ and $T \nsubseteq S$, the game is strictly convex. 
Ichiishi (1981) showed that a game $v$ is convex if and only if it satisfies the increasing marginal contributions (imc) inequalities

$$
v(T \cup\{i\})-v(T) \geq v(S \cup\{i\})-v(S)
$$

for all $S \subseteq T$ and $i \notin T$. So, not all inequalities in (*) are needed, and (**) suffices. However, also the system $(* *)$ has redundant inequalities, as will become apparent later on in this paper.

One of the main results on convex games is the Shapley-Ichiishi theorem. It can be stated as follows. Let $v$ be a game. Consider for a given permutation $\sigma$ of the player set $N$ the corresponding marginal vector $m^{\sigma}(v)$ defined by

$$
m^{\sigma}(v)_{\sigma(k)}:=v\left(S_{k}^{\sigma}\right)-v\left(S_{k-1}^{\sigma}\right)
$$

for each player $k \in N$, where $S_{0}^{\sigma}:=\varnothing$ and

$$
S_{k}^{\sigma}:=\{\sigma(j) \mid j \leq k\}
$$

for each $k \in\{1, \ldots n\}$. The result of Shapley (1971) and Ichiishi (1981) is

Theorem 1 The game $v$ is convex if and only if $m^{\sigma}(v) \in C(v)$ for each permutation $\sigma$.

For a convex game $v$, Theorem 1 implies that $\left\{S_{k}^{\sigma} \mid k \in N\right\} \subseteq T\left(v, m^{\sigma}\right)$ for each permutation $\sigma$. For a strictly convex game $v$, Shapley (1971) proved that $T\left(v, m^{\sigma}\right)=$ $\left\{S_{k}^{\sigma} \mid k \in N\right\}$ for each permutation $\sigma$. Hence, the core structure of a strictly convex game is given by

$$
\left\{\left\{S_{k}^{\sigma} \mid k \in N\right\} \mid \sigma \text { is a permutation of } N\right\}
$$

and every convex game is a limit game for a given strictly convex game.

\section{The facial structure of polyhedra}

Let $A$ be an $m \times n$ matrix and let $b \in \mathbb{R}^{m}$. The solution set

$$
P=\left\{x \in \mathbb{R}^{n} \mid A x \geq b\right\}
$$

of the system of linear inequalities $A x \geq b$ is a polyhedron. If $b=0$, then obviously $P$ is a cone, and $P$ is called a polyhedral cone. Evidently, every polyhedron is a convex set. The system $A x \geq b$ is a defining system for $P$.

The dimension of a convex subset $C$ of $\mathbb{R}^{n}$ is defined as the dimension of the smallest affine subspace that contains $C$. It is denoted by $\operatorname{dim}(C)$. A closed subset $F$ of $C$ is a face of $C$ if $x, y \in F$ if and only if $\frac{1}{2} x+\frac{1}{2} y \in F$. This definition implies that a face is convex. Thus each face has a dimension and a face with $\operatorname{dimension} \operatorname{dim}(C)-1$ is called a facet. A zero-dimensional face is called an extreme point. 
For polyhedra, there is an alternative way to describe faces. Denote the $i$-th row of $A$ by $a_{i}$, and let $I$ denote an index set of rows of $A$. Define

$$
F_{I}=\left\{x \in P \mid a_{i} x=b_{i} \text { for all } i \in I\right\} .
$$

It is easy to prove that each $F_{I}$ is a face of $P$, and conversely (see e.g. Schrijver 1986) each face of $P$ is of the type $F_{I}$. The polyhedron $P$ itself is obtained when we adopt the convention $P_{\emptyset}=P$. Every extreme point of $P$ can be obtained from an index set $I$, such that the rows $a_{i}$ for $i \in I$ form a basis of $\mathbb{R}^{n}$. Two extreme points are neighbors if there exist bases for them, such that the intersection of the linear subspaces spanned by the two bases has dimension $n-1$. The definition implies that also the line-segment between two neighboring extreme points is a face. Generically, this is a face of dimension 1, called edge. In the degenerate case, the two extreme points coincide, which happens if one extreme point is determined by two neighboring bases. In case $P$ is of full dimension, the facets are of dimension $n-1$, and each facet can be described by one inequality only. Therefore, if $P$ is of full dimension, the system where the inequalities correspond one-to-one to the facets is the unique minimal defining system for $P$, up to multiplication of the inequalities by positive scalars.

Notice that the system of inequalities $(*)$ that defines the class of convex games is of the type $A x \geq 0$. Hence the class of convex games is a polyhedral cone in the vector space of games. Any strictly convex game is obviously in the interior of this cone, so this cone is of full dimension. (Existence of a strictly convex game was demonstrated by Shapley (1971), and this paper contains an example in Sect. 5.)

Also the core of a game $v$ is obviously a polyhedron in $\mathbb{R}^{N}$, as the expression $x(S)$ for $S \subseteq N$ can be written as the linear expression $\left\langle e_{S}, x\right\rangle$, where $e_{S}$ is the vector with ones at the coordinates of $S$ and zeros elsewhere. We will abuse our terminology a bit, and say that a collection $B$ of coalitions is a basis if $\left\{e_{S} \mid S \in B\right\}$ is a basis for $\mathbb{R}^{n}$.

\section{Generalization of the Shapley-Ichiishi result}

From Theorem 1 it follows that the set of limit games $L(v)$ for a strictly convex game $v$ is the polyhedral cone of all convex games. In this section, we prove that the set $L(v)$ is a polyhedral cone for every balanced game $v$. In particular, we will describe how, given $v$, we can derive a system of linear inequalities whose solution set equals the polyhedral cone $L(v)$.

Let $v$ be a balanced game. We say that a basis $B$ of coalitions is feasible for $v$ if there is an extreme point $x \in C(v)$ such that $B \subseteq T(v, x)$. We say that a coalition $T \subseteq N$ is a neighbor of $B$ if there exists a feasible basis $C$ for $v$ such that $T \in C$ and $|B \cap C|=n-1$. We say that $T$ is spanned by $B$ if the unique numbers $\lambda_{S}$ for $S \in B$ to write

$$
e_{T}=\sum_{S \in B} \lambda_{S} e_{S}
$$

are nonnegative for all $S \in B \backslash\{N\}$. 
Fig. 1 Core of the game $v$

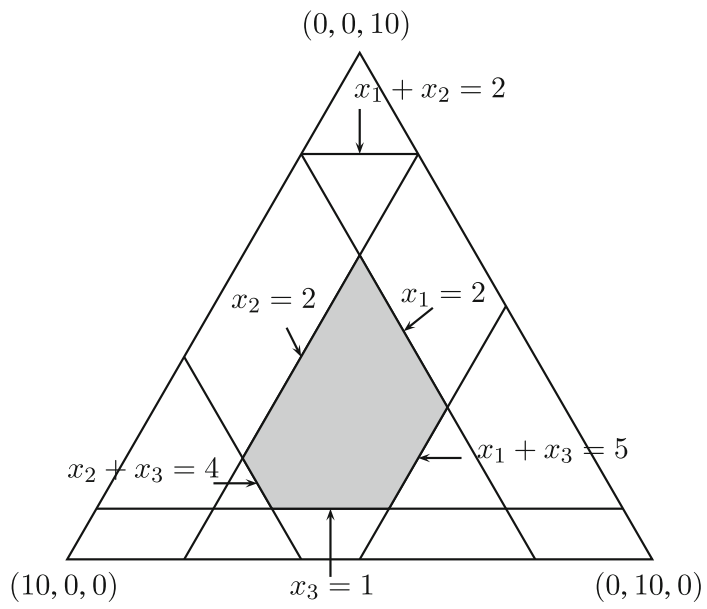

To illustrate these concepts, consider the following example. Let $v$ be the threeplayer game defined by

$$
v(S)= \begin{cases}2 & \text { if } S=\{1\} \text { or } S=\{2\} \text { or } S=\{1,2\} \\ 1 & \text { if } S=\{3\} \\ 5 & \text { if } S=\{1,3\} \\ 4 & \text { if } S=\{2,3\} \\ 10 & \text { if } S=N\end{cases}
$$

Straightforward calculation shows that the five extreme points of $C(v)$ are

$$
\begin{array}{ll}
a=(2,2,6) & T(v, a)=\{\{1\},\{2\},\{1,2,3\}\} \\
b=(2,5,3) & T(v, b)=\{\{1\},\{1,3\},\{1,2,3\}\} \\
c=(4,5,1) & \text { and that } \quad T(v, c)=\{\{3\},\{1,3\},\{1,2,3\}\} \\
d=(6,3,1) & T(v, d)=\{\{3\},\{2,3\},\{1,2,3\}\} \\
e=(6,2,2) & T(v, e)=\{\{2\},\{2,3\},\{1,2,3\}\} .
\end{array}
$$

Now, for example, coalition $\{1,3\}$ is a neighbor of $T(v, a)$, since $\{1,3\} \in T(v, b)$, and $a$ and $b$ are neighboring extreme points. The coalition $\{1,2\}$ is spanned by $T(v, a)$, since $e_{\{1,2\}}=e_{\{1\}}+e_{\{2\}}$. See also Fig. 1 .

Now, given the balanced game $v$, we construct a system of linear inequalities whose solution set equals the polyhedral cone $L(v)$. Let $B$ be a feasible basis and let $T \subseteq N$ be a neighbor of $B$ or spanned by $B$. Let $\lambda(S)$ for $S \in B$ be the unique real numbers such that

$$
e_{T}=\sum_{S \in B} \lambda(S) e_{S}
$$


The linear inequality generated by the pair $B, T$ is

$$
w(T) \leq \sum_{S \in B} \lambda(S) w(S),
$$

and we denote the set of all inequalities generated in this way by $I(v)$. Our claim is that $I(v)$ is a defining set for $L(v)$. In order to prove this claim we establish some preliminary facts.

Lemma 2 Let $v$ be a balanced game. If $T \in T(v, x) \backslash B$, where $x$ is an extreme point of $C(v)$ and $B \subseteq T(v, x)$ is a basis, then the inequalities in $I(v)$ imply

$$
w(T)=\sum_{S \in B} \lambda(S) w(S) .
$$

Proof Let $x, B$, and $T$ be as above. Since $T$ is a neighbor of $B, I(v)$ contains the inequality

$$
w(T) \leq \sum_{S \in B} \lambda(S) w(S)
$$

We show that the converse inequality is implied by the inequalities in $I(v)$. Consider the expression

$$
e_{T}=\sum_{S \in B} \lambda(S) e_{S}
$$

There is at least one coalition $U \in B$ with $\lambda(U)>0$. The collection $D:=(B \backslash\{U\}) \cup$ $\{T\}$ has exactly $n$ elements and $D$ is independent. Indeed, if $D$ were not independent, then either $B$ could not be a basis, or we could write $e_{T}$ as a linear combination of the elements $e_{S}$ with $S \in B$ without using $e_{U}$, which would violate the uniqueness of the coefficients. Consequently, $D$ is a basis and $U$ is a neighbor of $D$. Since

$$
e_{U}=\frac{1}{\lambda(U)} e_{T}-\sum_{\substack{S \in B \\ S \neq U}} \frac{\lambda(S)}{\lambda(U)} e_{S},
$$

the inequality

$$
w(U) \leq \frac{1}{\lambda(U)} w(T)-\sum_{\substack{S \in B \\ S \neq U}} \frac{\lambda(S)}{\lambda(U)} w(S)
$$

is in $I(v)$. And since $\lambda(U)>0$, this can be rewritten to

$$
w(T) \geq \sum_{S \in B} \lambda(S) w(S),
$$

which proves our claim. 
For a game $w$ that satisfies the inequalities in $I(v)$, we prove that $w$ is a limit game for $v$. If $x$ is an extreme point of $C(v)$ and $B$ a basis for $x$, then the solution $z$ to the system $y(S)=w(S)$ for all $S \in B$ is the obvious candidate for $z \in C(w)$ such that $T(v, x) \subseteq T(w, z)$. In the following lemma, we prove first that $z$ indeed satisfies the requirement $T(v, x) \subseteq T(w, z)$, regardless whether $z \in C(w)$ or not.

Lemma 3 Let $v$ be a balanced game and let $w$ be a game that satisfies the inequalities in $I(v)$. Let $x$ be an extreme point in $C(v)$ and let $B$ be a basis in $T(v, x)$. Let $z$ be the unique solution to the system of equalities

$$
y(S)=w(S) \quad \text { for all } S \in B .
$$

Then $T(v, x) \subseteq T(w, z)$.

Proof Let $T \in T(v, x)$. We show that $T \in T(w, z)$. This is trivial if $T \in B$, so suppose that $T \notin B$. Write

$$
e_{T}=\sum_{S \in B} \lambda(S) e_{S}
$$

By Lemma 2, the game $w$ satisfies the equality

$$
w(T)=\sum_{S \in B} \lambda(S) w(S)
$$

hence

$$
z(T)=\sum_{S \in B} \lambda(S) z(S)=\sum_{S \in B} \lambda(S) w(S)=w(T)
$$

which proves our claim.

A geometrical interpretation of the following lemma is that, if $w$ satisfies the system $I(v)$, the edges of $C(w)$ are parallel to those of $C(v)$, assuming that the candidate extreme points are indeed in $C(w)$.

Lemma 4 Let $v$ be a balanced game, and let $w$ be a game that satisfies all inequalities in $I(v)$. Let $x$ and $\bar{x}$ be neighboring extreme points of $C(v)$, with feasible basis $B$ and $\bar{B}$ respectively, such that $|B \cap \bar{B}|=n-1$. Let $z, \bar{z}$ be defined respectively as the unique solution of

$$
y(S)=w(S) \quad \text { for all } S \in B
$$

and

$$
y(S)=w(S) \quad \text { for all } S \in \bar{B}
$$


Then there exists $\mu \geq 0$ such that

$$
\bar{z}-z=\mu(\bar{x}-x) .
$$

Proof First assume that $\bar{x}=x$. Then $\bar{z}=z$ by Lemma 3 and we can set $\mu=0$. Now assume that $\bar{x} \neq x$. Write, for each $\alpha \in \mathbb{R}$,

$$
z(\alpha)=z+\alpha(\bar{x}-x)
$$

Let $T$ be the unique coalition in $\bar{B} \backslash B$ and write

$$
e_{T}=\sum_{S \in B} \lambda(S) e_{S}
$$

Since $w$ satisfies all inequalities in $I(v)$ and $T$ is a neighbor of $B$, in particular it satisfies the inequality

$$
w(T) \leq \sum_{S \in B} \lambda(S) w(S)
$$

Thus,

$$
z(T)=\sum_{S \in B} \lambda(S) z(S)=\sum_{S \in B} \lambda(S) w(S) \geq w(T) .
$$

Furthermore, since $\bar{x} \neq x, T$ is not tight at $x$ and hence $\left\langle e_{T}, \bar{x}-x\right\rangle<0$. This observation and the fact that $z(T) \geq w(T)$ implies that

$$
\hat{\alpha}:=\max \{\alpha \mid z(\alpha)(T) \geq w(T)\}
$$

exists and is not negative. From the definition of $z(\alpha)$ it is now clear that we can set $\mu=\hat{\alpha}$ if we can show $z(\hat{\alpha})=\bar{z}$.

To see why indeed $z(\hat{\alpha})=\bar{z}$, notice that all coalitions $S \in B \cap \bar{B}$ are tight at all $z(\alpha)$ in the game $w$, because they are tight at $z$ in $w$, and at both $x$ and $\bar{x}$ in $v$. Moreover, $T$ is tight at $z(\hat{\alpha})$ in $w$ by the definition of $\hat{\alpha}$. So, all coalitions in $\bar{B}$ are tight at $z(\hat{\alpha})$ in $w$. Hence, $z(\hat{\alpha})=\bar{z}$.

We have now developed enough equipment to prove the main theorem, namely that the system of inequalities in $I(v)$ precisely defines the set $L(v)$, which must therefore be a polyhedral cone.

Theorem 5 Let $v$ be a balanced game. Then a game $w$ satisfies the inequalities in $I(v)$ if and only if $w \in L(v)$.

Proof A. Let $w \in L(v)$. We show that $w$ satisfies the inequalities in $I(v)$. To this end, let $B$ be a feasible basis for $v$, let $T \subseteq N$, and let $\lambda(S)$ for $S \in B$ be real numbers such that 


$$
e_{T}=\sum_{S \in B} \lambda(S) e_{S}
$$

Since $w$ is a limit game for $v$, we can choose an extreme point $z \in C(w)$ such that $T(v, x) \subseteq T(w, z)$. Then

$$
w(T) \leq z(T)=\left\langle z, e_{T}\right\rangle=\sum_{S \in B} \lambda(S) z(S)=\sum_{S \in B} \lambda(S) w(S)
$$

This proves that $w$ satisfies all inequalities in $I(v)$. Since we did not use the fact that $T$ is a neighbor of $B$ or spanned by $B$, in fact we proved that $w$ satisfies many more inequalities.

B. Now suppose that $w$ satisfies all inequalities in $I(v)$. Let $x$ be an extreme point of $C(v)$. We need to prove that there exists a $z \in C(w)$ such that $T(v, x) \subseteq T(w, z)$. To this end, let $B \subseteq T(v, x)$ be a basis, and define $z$ as the unique solution of the system

$$
y(S)=w(S) \quad \text { for all } S \in B
$$

By Lemma 3, $T(v, x) \subseteq T(w, z)$, so it remains to prove that $z \in C(w)$.

First note that $z(N)=w(N)$, since $N \in T(v, x) \subseteq T(w, z)$. Now let $T \neq N$. We need to show that $z(T) \geq w(T)$. Consider the program

$$
\mathcal{P}: \min \left\langle e_{T}, y\right\rangle \text { s.t. } y \in C(v)
$$

Solving this linear program by means of the simplex algorithm using starting point $y^{1}=x$ and basis $B_{1}=B$ for the initialization, yields a sequence

$$
x=y^{1}, \ldots, y^{k}=: \bar{x}
$$

of extreme points of $C(v)$ together with a basis $B_{m}$ for each point $y^{m}$. From the properties of the simplex algorithm we have

$$
\begin{aligned}
& \left|B_{m+1} \cap B_{m}\right|=n-1 \\
& \left\langle e_{T}, y^{m+1}-y^{m}\right\rangle \leq 0 .
\end{aligned}
$$

Now, for each $B_{m}$, let $z^{m}$ be the unique solution to the system

$$
y(S)=w(S) \quad \text { for all } S \in B_{m}
$$

of linear equalities, and let $\bar{z}=z^{k}$. We will prove that $z(T) \geq \bar{z}(T) \geq w(T)$.

By Lemma 4, there exists nonnegative numbers $\mu_{m}$, such that $z^{m+1}-z^{m}=$ $\mu_{m}\left(y^{m+1}-y^{m}\right)$ for $m \in\{1, \ldots, k\}$. Hence, 


$$
\begin{aligned}
\bar{z}(T)=z^{k}(T)=\left\langle e_{S}, z^{k}\right\rangle & =\left\langle e_{T}, z^{1}\right\rangle+\sum_{m=1}^{k-1}\left\langle e_{T}, z^{m+1}-z^{m}\right\rangle \\
& =z^{1}(T)+\sum_{m=1}^{k-1} \mu_{m}\left\langle e_{T}, y^{m+1}-y^{m}\right\rangle \\
& \leq z^{1}(T)=z(T) .
\end{aligned}
$$

It remains to prove that $\bar{z}(T) \geq w(T)$. If $T \in T(v, \bar{x})$, then also $T \in T(w, \bar{z})$, hence $\bar{z}(T)=w(T)$. Otherwise, consider the dual of the linear program $\mathcal{P}$, given by

$$
\begin{array}{cl}
\min & \sum_{S \neq \emptyset} \alpha_{S} v(S) \\
\mathcal{D}: \quad \text { subject to } & \sum_{S \neq \emptyset} \alpha_{S} e_{S}=e_{T} \\
& \alpha_{S} \geq 0 \quad \text { for all } S \neq \emptyset, N .
\end{array}
$$

Let $\lambda_{S}(S \neq \emptyset)$ be an extreme optimal solution of the dual, and let $B$ be the collection of coalitions $S$ for which $\lambda_{S} \neq 0$. By the complementary slackness relations of linear programming, we have $\bar{y}(S)=v(S)$ for all $S$ with $\lambda_{S} \neq 0$, hence $B \subseteq T(v, \bar{x})$. Since $\lambda$ was chosen extreme, $B$ is independent and contains at most $n$ coalitions. If $B$ is not already a basis, then we extend it to a basis with coalitions from $T(v, \bar{x})$. Then

$$
e_{T}=\sum_{S \in B} \lambda_{S} e_{S}
$$

with $\lambda_{S} \geq 0$ for all $S \in B \backslash\{N\}$, since $\lambda$ is feasible for $\mathcal{D}$. We see that $T$ is spanned by $B$, and since $w$ satisfies all inequalities in $I(v)$, it satisfies in particular the inequality

$$
w(T) \leq \sum_{S \in B} \lambda_{S} w(S)
$$

Then

$$
w(T) \leq \sum_{S \in B} \lambda_{S} w(S)=\sum_{S \in B} \lambda_{S} \bar{z}(S)=\bar{z}(T)
$$

In the definition of $I(v)$, we tried to economize on the number of inequalities as much as possible. In case the polyhedral cone $L(v)$ defined by $I(v)$ is of full dimension, one might hope that $I(v)$ is the unique minimal defining system for it. We conclude this section with an example to show that this is not true in general. Consider the following game with player set $N=\{1,2,3,4\}$. 
Fig. 2 Neighboring extreme points of core $(v)$

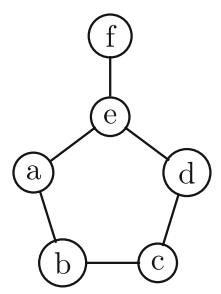

$$
v(S)=\left\{\begin{aligned}
0 & \text { if } S=\{1\} \text { or } S=\{4\} \\
2 & \text { if } S=\{2\} \text { or } S=\{3\} \\
5 & \text { if } S=\{1,2\} \text { or } S=\{1,3\} \text { or } S=\{2,3\} \\
-1 & \text { if } S=\{1,4\} \text { or } S=\{2,4\} \text { or } S=\{3,4\} \\
-1 & \text { if }|S|=3 \text { and } 4 \in S \\
9 & \text { if } S=\{1,2,3\} \\
11 & \text { if } S=N
\end{aligned}\right.
$$

With some effort, one can verify that $C(v)$ has twelve extreme points. These are

$$
\begin{array}{ll}
a=(3,4,2,2) & g=(0,5,6,0) \\
b=(4,3,2,2) & h=(0,6,5,0) \\
c=(4,2,3,2) & i=(3,2,6,0) \\
d=(3,2,4,2) & j=(3,6,2,0) \\
e=(1,4,4,2) & k=(6,2,3,0) \\
f=(0,5,5,1) & l=(6,3,2,0) .
\end{array}
$$

For the twelve extreme core elements above we can check that

$$
\begin{array}{ll}
T(v, a)=\{\{3\},\{1,3\},\{1,2,3\}, N\} & T(v, g)=\{\{1\},\{4\},\{1,2\}, N\} \\
T(v, b)=\{\{3\},\{2,3\},\{1,2,3\}, N\} & T(v, h)=\{\{1\},\{4\},\{1,3\}, N\} \\
T(v, c)=\{\{2\},\{2,3\},\{1,2,3\}, N\} & T(v, i)=\{\{2\},\{4\},\{1,2\}, N\} \\
T(v, d)=\{\{2\},\{1,2\},\{1,2,3\}, N\} & T(v, j)=\{\{3\},\{4\},\{1,3\}, N\} \\
T(v, e)=\{\{1,2\},\{1,3\},\{1,2,3\}, N\} & T(v, k)=\{\{2\},\{4\},\{2,3\}, N\} \\
T(v, f)=\{\{1\},\{1,2\},\{1,3\}, N\} & T(v, l)=\{\{3\},\{4\},\{2,3\}, N\} .
\end{array}
$$

Notice that every collection of tight coalitions is a basis. It is then straightforward to prove that, for small perturbations of $v$, the core structure remains the same. Hence, the cone $L(v)$ is of full dimension, and has a unique minimal defining system. The defining system $I(v)$ however has redundant inequalities in it. To see this, consider the graph of neighboring extreme points, restricted to the six points $a, b, c, d, e$ and $f$ (Fig. 2).

Each edge in this graph induces an inequality. For example, the inequality $(f \rightarrow e)$ is generated by writing $e_{\{1,2,3\}}$ as a linear combination of the characteristic vectors corresponding to the elements in $T(v, f)$ :

$$
e_{\{1,2,3\}}=e_{\{1,2\}}+e_{\{1,3\}}-e_{\{1\}} \text {. }
$$


The inequalities thus induced by the edges in this graph are

$$
\begin{array}{ll}
w(\{1,2,3\}) \leq w(\{1,2\})+w(\{1,3\})-w(\{1\}) & (f \rightarrow e) \\
w(\{3\}) \leq w(\{1,2,3\})-w(\{1,2\}) & (e \rightarrow a) \\
w(\{2,3\}) \leq w(\{1,2,3\})+w(\{3\})-w(\{1,3\}) & (a \rightarrow b) \\
w(\{2\}) \leq w(\{2,3\})-w(\{3\}) & (b \rightarrow c) \\
w(\{1,2\}) \leq w(\{1,2,3\})-w(\{2,3\})+w(\{2\}) & (c \rightarrow d) \\
w(\{1,3\}) \leq w(\{1,2,3\})-w(\{2\}) & (d \rightarrow e) .
\end{array}
$$

Notice that the inequality $(e \rightarrow a)$ is implied by inequalities $(b \rightarrow c)$ and $(c \rightarrow d)$. Symmetrically, $(d \rightarrow e)$ is implied by $(a \rightarrow b)$ and $(b \rightarrow c)$.

\section{Application to convex games}

In this section, we investigate the consequences of Theorem 5, when it is applied to a strictly convex game. One could use Shapley's (1971) characterization of the core structure of these games, as described after Theorem 1, and apply Theorem 5 directly. We do not wish to rely on Shapley's result however. We will specify a strictly convex game and go through the whole process. Shapley's result makes clear that the result will be independent of our specific choice.

Define $v^{*}$ by

$$
v^{*}(S):=s^{2}
$$

for each coalition $S$, where $s=|S|$. Then it is straightforward to prove that

$$
v(S \cup T)+v(S \cap T)=v(S)+v(T)+2 a b,
$$

where $a=|S \backslash T|$ and $b=|T \backslash S|$. Since $2 a b \geq 0$, it follows that $v^{*}$ satisfies the system $(*)$. Indeed, $v^{*}$ is strictly convex, since equality in (*) only occurs if $a=0$ or $b=0$, i.e. if $S \subseteq T$ or $T \subseteq S$.

We now establish the core structure of $v^{*}$.

Lemma 6 The convex hull of the marginal vectors of $v^{*}$ equals $C\left(v^{*}\right)$. Moreover, $T\left(v^{*}, m^{\sigma}\left(v^{*}\right)\right)=\left\{S_{k}^{\sigma} \mid k \in N\right\}$ for each marginal vector $m^{\sigma}\left(v^{*}\right)$.

Proof Let $m^{\sigma}\left(v^{*}\right)$ be a marginal vector of $v^{*}$. Assume w.l.o.g. that $\sigma$ is the identity. Thus, the payoff to player $i$ equals $i^{2}-(i-1)^{2}=2 i-1$, and for a (non-empty) coalition $S$ with $|S|=s$ we get that

$$
m^{\sigma}\left(v^{*}\right)(S)=\sum_{i \in S}(2 i-1) \geq \sum_{i=1}^{s}(2 i-1)=2 \cdot \frac{1}{2} s(s+1)-s=s^{2}=v^{*}(S) .
$$

Let $x$ be an extreme point of $C\left(v^{*}\right)$. To see that $T\left(v^{*}, x\right)=\left\{S_{k}^{\sigma} \mid k \in N\right\}$ for a suitably chosen permutation $\sigma$, it suffices to show that for any $S, T \subseteq N$ that are both tight at $x$ in $v^{*}$, either $S \subseteq T$ or $T \subseteq S$. Note that 
$v^{*}(S \cap T)+v^{*}(S \cup T) \leq x(S \cap T)+x(S \cup T)=x(S)+x(T)=v^{*}(S)+v^{*}(T)$.

So, by the convexity of $v^{*}, v^{*}(S \cap T)+v^{*}(S \cup T)=v^{*}(S)+v^{*}(T)$. It follows that $S \subseteq T$ or $T \subseteq S$ because $v^{*}$ is strictly convex.

Theorem 7 The system $I\left(v^{*}\right)$ is the system of inequalities

$$
v(S \cup\{i, j\})-v(S \cup\{j\}) \geq v(S \cup\{i\})-v(S), \quad(* * *)
$$

for all $i, j \in N(i \neq j)$ and all $S \subseteq N \backslash\{i, j\}$.

Proof Let $S, i, j$ be such that $S \subseteq N \backslash\{i, j\}$ and $i \neq j$. Let $\tau$ be a permutation for which the elements of $S$ come first, then $i$, then $j$, and then the elements of $N \backslash(S \cup\{i, j\})$. Let $\sigma$ be the permutation for which the elements of $S$ are ordered as in $\tau$, then $j$, then $i$, and then the elements of $N \backslash(S \cup\{i, j\})$ as in $\tau$. Note that $S \cup\{j\}$ is a neighbor of $T\left(v^{*}, m^{\tau}\left(v^{*}\right)\right)$ and that $S, S \cup\{j\}, S \cup\{i, j\} \in T\left(v^{*}, m^{\tau}\left(v^{*}\right)\right)$. Thus, since

$$
e_{T}=e_{S \cup\{j\}}=e_{S \cup\{i, j\}}-e_{S \cup\{i\}}+e_{S}
$$

(where $e_{S}$ denotes the zero vector when $S$ is empty), the inequality in $I\left(v^{*}\right)$ generated by the choice of $S, i, j$ is

$$
w(S \cup\{j\}) \leq w(S \cup\{i, j\})-w(S \cup\{i\})+w(S),
$$

which can be rewritten as

$$
w(S \cup\{i, j\})-w(S \cup\{j\}) \geq w(S \cup\{i\})-w(S)
$$

So indeed, all inequalities in $(* * *)$ are generated.

To see that no other inequalities are generated, associated with permutations $\sigma, \tau$, note that the requirement $\left|T\left(v^{*}, m^{\sigma}\left(v^{*}\right)\right) \cap T\left(v^{*}, m^{\tau}\left(v^{*}\right)\right)\right|=n-1$ implies that $\tau$ can be obtained from $\sigma$ by a switch of two players $i, j$, with $j$ directly after $i$ in $\sigma$. Then let $S$ be the set of players that come before $i$ in $\sigma$, and it is evident that $\sigma$ and $\tau$ generate the inequality corresponding to $S, i, j$.

We obtain the following variant of the Shapley-Ichiishi result as a corollary.

Corollary 8 A game $v$ satisfies the inequalities in $(* * *)$ if and only if all marginal vectors are elements of $C(v)$.

Proof Suppose that $v$ satisfies the inequalities in $(* * *)$. Then $v$ is a limit game for $v^{*}$, by Theorem 5. Hence, $m^{\sigma}(v)$ is an extreme point of $C(v)$ for every $\sigma$.

Suppose that $m^{\sigma}(v) \in C(v)$ for every $\sigma$. Then note that every $m^{\sigma}(v)$ is extreme in $C(v)$, so it follows that $v$ is a limit game for $v^{*}$. By Theorem 5, $v$ satisfies the inequalities in $I\left(v^{*}\right)$, and by Theorem 7 , this is the system $(* * *)$. 
Note that the set of inequalities $(* * *)$ is the set $I(v)$ for any strictly convex game $v$. Hence, it also follows immediately that a game is convex precisely when it is a limit game of any strictly convex game.

Corollary 8 characterizes the games for which all marginal vectors are in the core with less inequalities than it is usually done. So, as a side result we proved that the system $(* * *)$ implies the systems $(* *)$ and $(*)$.

System $(* * *)$, which we derived in Sect. 5, is in fact the unique minimal representation for the polyhedral cone of convex games, up to multiplication of the inequalities by positive scalars. To prove that each inequality in $(* * *)$ indeed describes a facet of this cone, we will demonstrate that for each inequality in $I(v)$, there exist a game that violates the inequality, but satisfies all other inequalities in $I(v)$. In the proof, we make use of an alternative characterization of convex games by means of unanimity games.

A convenient basis for the vector space of all games was introduced in Shapley (1953). Let $T$ be a non-empty coalition in the player set $N$. The corresponding unanimity game $u_{T}$ is defined as follows.

$$
u_{T}(S)= \begin{cases}1 & \text { if } T \subseteq S \\ 0 & \text { otherwise }\end{cases}
$$

Shapley (1953) proved that the collection of unanimity games forms a basis of the vector space of all games. In other words, given a game $v$, there exist unique coefficients $\left(\alpha_{T}\right)_{T \neq \phi}$ such that

$$
v=\sum_{T \neq \emptyset} \alpha_{T} u_{T}
$$

Many classes of games, like airport games (Littlechild and Owen 1973) and sequencing games (Curiel et al. 1989), can be characterized through restrictions on these coefficients. Also the class of convex games can be characterized through those coefficients.

Theorem 9 The following two conditions are equivalent:

(a) The game $v=\sum_{T \neq \emptyset} \alpha_{T} u_{T}$ is convex.

(b) For all triplets $(S, i, j)$ with $i \neq j$ and $i, j \notin S$ :

$$
\sum_{T \subseteq S} \alpha_{T \cup\{i, j\}} \geq 0,
$$

where the numbers $\alpha_{T}$ are the coefficients of the game $v$ with respect to the basis of unanimity games.

Proof By Corollary 8 it suffices to prove the equivalence of $(* * *)$ and (b). Write $v=\sum_{T \neq \emptyset} \alpha_{T} u_{T}$. Let $i, j \in N$ such that $i \neq j$, and let $S \subseteq N \backslash\{i, j\}$. Then 


$$
\begin{gathered}
v(S \cup\{i, j\})-v(S \cup\{j\}) \geq v(S \cup\{i\})-v(S) \\
\Leftrightarrow \\
{[v(S \cup\{i, j\})-v(S \cup\{j\})]-[v(S \cup\{i\})-v(S)] \geq 0} \\
\Leftrightarrow \\
{\left[\sum_{T \subseteq S \cup\{i, j\}} \alpha_{T}-\sum_{T \subseteq S \cup\{j\}} \alpha_{T}\right]-\left[\sum_{T \subseteq S \cup\{i\}} \alpha_{T}-\sum_{T \subseteq S} \alpha_{T}\right] \geq 0} \\
\Leftrightarrow \\
\sum_{T \subseteq S \cup\{j\}} \alpha_{T \cup\{i\}}-\sum_{T \subseteq S} \alpha_{T \cup\{i\}} \geq 0 \\
\Leftrightarrow \\
\sum_{T \subseteq S} \alpha_{T \cup\{i, j\}} \geq 0 .
\end{gathered}
$$

Lemma 10 Let $i, j \in N$ with $i \neq j$ and let $S \subseteq N \backslash\{i, j\}$. Let $v=\sum_{T} \alpha_{T} u_{T}$ with

$$
\alpha_{T}=\left\{\begin{aligned}
-1 & \text { if } T=S \cup\{i, j\} \\
1 & \text { if } T=S \cup\{i\} \text { or } T=S \cup\{j\} \text { or } T \nsubseteq S \cup\{i, j\} \\
0 & \text { otherwise. }
\end{aligned}\right.
$$

Then $v$ violates the inequality

$$
v(S \cup\{i, j\})-v(S \cup\{j\}) \geq v(S \cup\{i\})-v(S)
$$

and satisfies all other inequalities in $(* * *)$.

Proof Notice that $\sum_{T \subset S} \alpha_{T \cup\{i, j\}}=-1<0$, so by Theorem 9, the condition for $(S, i, j)$ is indeed violated. To show that all other inequalities are satisfied, let $k, l \in$ $N, k \neq l$ and $V \subseteq N \backslash\{k, l\}$, such that the triplet $(V, k, l) \neq(S, i, j)$. Consider the quantity

$$
\sum_{T \subseteq V} \alpha_{T \cup\{k, l\}}
$$

If $\alpha_{S \cup\{i, j\}}$ does not appear in the sum, then it is a sum of only nonnegative terms, hence it is nonnegative. If $\alpha_{S \cup\{i, j\}}$ does appear in the summation, discern two cases.

(a) $V \neq S$ and $\{i, j\}=\{k, l\}$. The fact that $\alpha_{S \cup\{i, j\}}$ appears in the above sum implies that the set $S$ is a subset of $V$. So, since $V \neq S$, we can choose $m \in V \backslash S$. Then $\alpha_{\{m\} \cup\{k, l\}}=1$ appears in the sum too, compensating for $\alpha_{S \cup\{i, j\}}=-1$ and consequently yielding a nonnegative outcome.

(b) $\{i, j\} \neq\{k, l\}$. Assume without loss of generality that $i \notin\{k, l\}$. Since $\alpha_{S \cup\{i, j\}}=$ -1 appears in the sum, also $\alpha_{S \cup\{j\}}=1$ appears in it, compensating the negative number and hence yielding a nonnegative outcome.

Corollary 11 The system $(* * *)$ is a facet description for the class of convex games.

Proof Since $L\left(v^{*}\right)$ is of full dimension, i.e., of dimension $2^{n}-1$, we can choose a full-dimensional ball $B$ of strictly convex games. Let $v$ be a game that violates the 
inequality associated with $(S, i, j)$ and none of the other inequalities in $(* * *)$. Let $K$ be the convex hull of $v$ and the ball $B$. Let $L$ be the intersection of $K$ and the hyperplane $w(S \cup\{i, j\})-w(S \cup\{j\})=w(S \cup\{i\})-w(S)$. It is obvious that $L$ consists of convex games only, hence it a subset of the face determined by $(S, i, j)$. It is also obvious that $L$ has dimension $2^{n}-2$, so it follows that $(S, i, j)$ determines a face of dimension $2^{n}-2$, hence a facet.

We conclude with a brief remark on the complexity of a minimal test for convexity. There are $\left(\begin{array}{l}n \\ 2\end{array}\right)$ ways to choose two different players $i$ and $j$ in $N$, and $2^{n-2}$ ways to choose a coalition $S$ that does not contain players $i$ and $j$. Thus we have $2^{n-2}\left(\begin{array}{l}n \\ 2\end{array}\right)$ inequalities of the form

$$
v(S \cup\{i, j\})-v(S \cup\{j\}) \geq v(S \cup\{i\})-v(S) .
$$

Since the input size of a game is $x=2^{n}-1$ (i.e. the number of nonempty coalitions), the complexity of testing all inequalities in $(* * *)$ is $\mathcal{O}\left(x\left(\log _{2} x\right)^{2}\right)$. Testing the original system $(*)$ has complexity $\mathcal{O}\left(x^{2}\right)$, and testing the system $(* *)$ of Ichiishi has complexity $\mathcal{O}\left(x^{\log _{2} 3} \log _{2} x\right)$.

\section{Conclusion}

In this paper, we presented a generalization of the Shapley-Ichiishi result to arbitrary balanced games. We introduced an equivalence relation on the class of balanced games, based on the notion of core structure. We showed how, given an arbitrary balanced game, we can construct a system of linear inequalities that describes the polyhedral cone of limits of games that are equivalent to the game under consideration. We also showed how the classical result of Shapley and Ichiishi on convex games can be obtained as a special case of our result. The defining system of linear inequalities presented in our main theorem is shown to be minimal in the special case of convex games. In an example we demonstrated that in general this is not necessarily the case. An interesting open question in this context is whether our procedure can be adapted in such a way (other than the obvious brute force method) that the resulting procedure always yields a minimal defining system.

As a final remark, we briefly discuss in more detail how our main theorem can be used to compute strategically stable sets of strategy pairs in bimatrix games. Hillas (1990) defined quasi-stable sets. A closely related notion, in fact quasi-stability minus the invariance and minimality requirements, is the notion of a Q-set, defined in Vermeulen et al. (1996). For a bimatrix game $(A, B)$, a Q-perturbed version of $(A, B)$ is a strategic form game in which the payoffs for the players are the same as in the original game, but each player is only allowed to select strategies from a-strictsubset of his strategy space. These subsets take the form of the core of a TU-game, which gives the connection to the current paper. A closed set $S$ of strategy pairs is a Q-set if every Q-perturbed version of $(A, B)$ has an equilibrium close to $S$. Vermeulen et al. (1996) showed that for bimatrix games, minimal Q-sets are finite.

Their approach also shows that, given a closed set $S$ of strategy pairs for a given bimatrix game, testing whether $S$ is a Q-set is a finite task. The reason for this is that 
many Q-perturbed versions of $(A, B)$ in fact impose the same conditions on $S$ for it to be a $\mathrm{Q}$-set. That way we can construct an equivalence relation on $\mathrm{Q}$-perturbed versions of $(A, B)$, by saying that two Q-perturbations are equivalent when they impose the same condition on $S$. It is shown in Vermeulen et al. (1996) that there are only finitely many equivalence classes under this equivalence relation.

Now, the strategy spaces of two equivalent Q-perturbations, viewed as cores of two TU-games, can be shown to have the same core structure. Thus, when devising a finite test to check whether a closed set $S$ is a Q-set, one needs to determine all classes of TU-games having the same core structure. The search for classes of TU-games having the same core structure becomes manageable when these classes are given by linear (in)equalities. And the task to describe all classes of TU-games having the same core structure by means of linear (in)equalities is executed in this paper. In that sense the current paper develops one of the tools needed to construct a finite test for Q-sets.

Acknowledgements Financial support from the Wallander/Hedelius Foundation is gratefully acknowledged. We thank two anonymous referees, William Thomson, and Rakesh Vohra for their helpful comments on earlier drafts of this paper, and Jean Derks for many fruitful discussions. Last but not least, we would like to thank Sofia Grahn for her help and contribution during the early stages of development of this paper.

Open Access This article is distributed under the terms of the Creative Commons Attribution Noncommercial License which permits any noncommercial use, distribution, and reproduction in any medium, provided the original author(s) and source are credited.

\section{References}

Aumann RJ, Maschler M (1985) Game theoretic analysis of a bankruptcy problem from the Talmud. J Econ Theory 36:195-213

Curiel I, Pederzoli G, Tijs S (1989) Sequencing games. Eur J Oper Res 40:344-351

Driessen TSH (1986) Contributions to the theory of cooperative games: the $\tau$-value and $k$-convex games. Ph.D Thesis, University of Nijmegen.

Granot D, Maschler M, Owen G, Zhu WR (1996) The kernel/nucleolus of a standard tree game. Int J Game Theory 25:219-244

Granot D, Kuipers J, Chopra S (2002) Cost allocation for a tree network with heterogeneous customers. Math Oper Res 27:647-661

Hillas J (1990) On the definition of the strategic stability of equilibria. Econometrica 58:1365-1390

Ichiishi T (1981) Supermodularity: applications to convex games and to the greedy algorithm for LP. J Econ Theory 25:283-286

Littlechild SC, Owen G (1973) A simple expression for the Shapley value in a special case. Manag Sci 20:370-372

Megiddo N (1978) Computational complexity of the game theory approach to cost allocation for a tree. Math Oper Res 3:189-196

O’Neill B (1982) A problem of rights arbitration from the Talmud. Math Soc Sci 2:345-371

Schrijver A (1986) Theory of linear and integer programming. Wiley, New York

Shapley LS (1953) A value for $n$-person games. In: Kuhn H, Tucker AW (eds) Contributions to the theory of games II. Princeton University Press, Princeton, pp 307-317

Shapley LS (1971) Cores of convex games. Int J Game Theory 1:11-26

Vermeulen AJ, Potters JAM, Jansen MJM (1996) On quasi-stable sets. Int J Game Theory 25:43-49 